\title{
Vitamin C in the Prevention of Postoperative Atrial Fibrillation: A New Challenge for Cardiothoracic Nurses
}

\section{Konstantinos Giakoumidakis}

Cardiac Surgery Intensive Care Unit "Evangelismos" General Hospital of Athens, Ipsilantou 45-47 Street, 10676, Athens, Greece

Atrial Fibrillation (AF) is the most common rhythm disturbance noted after open heart surgery procedures with a reported incidence of $10 \%$ to $65 \%$ depending on many factors, including patient history, preoperative medications and type of surgery [1]. The onset of AF usually occurs on the second or third postoperative day, is in most cases self-limited and $80 \%$ of patients return to sinus rhythm within one to three days following correction of electrolyte abnormalities and pharmaceutical treatment with $\beta$-blockers, amiodarone, calcium channel blockers or digoxin $[2,3]$.

AF is a major cause of morbidity and mortality in cardiac operated patients and has been associated with poor outcomes, such as hemodynamic compromise, ventricular arrhythmias, postoperative stroke, myocardial infarction, heart failure and significantly longer intensive care unit and hospital length of stay $[2,4]$. Despite the current advances in surgery, anesthesia, drug therapy and postoperative care, the incidence of AF arising de novo after cardiac surgery has not been decreased [3]. In addition, radiofrequency ablation has recently shown promising results but is an invasive procedure which is indicated only in selected patients where conventional antiarrhythmics have failed to provide symptomatic control [2].

Current evidence supports that the pathogenesis of postoperative $\mathrm{AF}$ is multifactorial, with inflammation and oxidative stress playing important roles [5]. In addition, it is well known that cardiac surgery is characterized by acute ischemic and reperfusion injury, which leads to the release of reactive oxygen species, causing oxidative stress and a systematic inflammatory response [6]. Therefore, it is reasonable to investigate the hypothesis that the prophylactic use of antioxidant vitamins, such as vitamin $\mathrm{C}$, could protect cardiac operated patients against oxidative stress and consequently against the onset of $\mathrm{AF}$ postoperatively.

The literature review reveals studies that have found significant correlation between the prophylactic therapy with ascorbic acid and the reduction of the incidence of postoperative AF among cardiac surgery patients. Sisto et al. [7] Stated that the incidence of postoperative arrhythmias, including AF, was lower in the patients who were treated with antioxidant vitamins, including vitamin $\mathrm{C}$ and allopurinol, relative to controls. In addition, as highlighted by the studies of Eslami et al. [8] and Papoulidis et al. [3] the combination of vitamin $\mathrm{C}$ and $\beta$-blockers may be more effective in reducing postoperative AF than $\beta$-blockers alone. However, other studies do not provide strong evidence regarding the preventive role of ascorbic acid in AF postoperatively occurrence [9].

Despite the methodological limitations of the above studies and the need for further research, based on high quality studies, the literature data highlighted the possible preventive effect of vitamin $\mathrm{C}$ in the occurrence of AF during the postoperative period of cardiac surgery patients. Clinicians have a new therapeutic option regarding the reduction of both the $\mathrm{AF}$ incidence and the poor outcomes that accompany this arrhythmia. In addition, cardio-thoracic nurses face new challenges in caring for cardiac surgery patients, which are not limited in the administration of these medications. Nurses should incorporate in the preoperative teaching of cardiac surgery patients' information regarding the duration, the dosage and the side effects of the antioxidant therapy with vitamin C. Also, cardiac surgery patients should be educated regarding the aim and the prophylactic effect of this treatment. Usually the administration of ascorbic acid begins a few hours or days prior to surgery (usually $2 \mathrm{~g}$ the night before surgery) and is being continued for 2-5 days after surgery (usually $500 \mathrm{mg}$ twice daily) [8,9]. Although, nausea, vomiting, headaches, heartburn, insomnia and diarrhea are among the bothersome adverse effects of ascorbic acid, this medication does not cause significant side effects in the above dosages.

In conclusion, the current link between oxidative stress and AF has led to new prophylactic approaches, which include the preoperative and postoperative administration of vitamin $\mathrm{C}$ among cardiac surgery patients. Based on findings of recent studies, this antioxidant intervention has an encouraging effect on postoperative AF incidence reduction. Cardio-thoracic nurses may play a significant role on cardiac surgery patient health promotion and rehabilitation, incorporating patient education regarding these agents in the holistic care of cardiac operated patients.

\section{References}

1. Kern LS (2004) Postoperative atrial fibrillation: new directions in prevention and treatment. J Cardiovasc Nurs 19: 103-115.

2. Rasoli S, Kakouros N, Harling L, Gukop P, Soni M, et al. (2011) Antioxidant vitamins in the prevention of atrial fibrillation: what is the evidence? Cardiol Res Pract 2011: 164078.

3. Papoulidis $P$, Ananiadou O, Chalvatzoulis E, Ampatzidou F, Koutsogiannidis $C$, et al. (2011) The role of ascorbic acid in the prevention of atrial fibrillation after elective on-pump myocardial revascularization surgery: a single-center experience--a pilot study. Interact Cardiovasc Thorac Surg 12: 121-124.

4. Keller KB, Lemberg $L(2008)$ The importance of vitamin $C$ in the incidence of atrial fibrillation. Am J Crit Care 17: 270-272.

5. Banach M, Kourliouros A, Reinhart KM, Benussi S, Mikhailidis DP, et al. (2010) Postoperative atrial fibrillation - what do we really know? Curr Vasc Pharmaco 8: 553-572.

6. Laffey JG, Boylan JF, Cheng DC (2002) The systemic inflammatory response to cardiac surgery: implications for the anesthesiologist. Anesthesiology 97 : 215-252.

7. Sisto T, Paajanen H, Metsä-Ketelä T, Harmoinen A, Nordback I, et al. (1995) Pretreatment with antioxidants and allopurinol diminishes cardiac onset events in coronary artery bypass grafting. Ann Thorac Surg 59: 1519-1523.

Corresponding author: Konstantinos Giakoumidakis, Cardiac Surgery Intensive Care Unit "Evangelismos" General Hospital of Athens, Ipsilantou 45-47 Street 10676, Athens, Greece, Tel: 00306973793489; Fax: 00302132041987; Email: kongiakoumidakis@gmail.com

Received May 21, 2012; Accepted May 23, 2012; Published May 25, 2012

Citation: Giakoumidakis K (2012) Vitamin C in the Prevention of Postoperative Atrial Fibrillation: A New Challenge for Cardiothoracic Nurses. J Nurs Care 1:e106. doi:10.4172/2167-1168.1000e106

Copyright: () 2012 Giakoumidakis K. This is an open-access article distributed under the terms of the Creative Commons Attribution License, which permits unrestricted use, distribution, and reproduction in any medium, provided the original author and source are credited. 
Citation: Giakoumidakis K (2012) Vitamin C in the Prevention of Postoperative Atrial Fibrillation: A New Challenge for Cardiothoracic Nurses. J Nurs Care 1:e106. doi:10.4172/2167-1168.1000e106

Page 2 of 2

8. Eslami M, Badkoubeh RS, Mousavi M, Radmehr H, Salehi M, et al (2007) Ora ascorbic acid in combination with beta-blockers is more effective than betablockers alone in the prevention of atrial fibrillation after coronary artery bypass grafting. Tex Heart Inst J 34: 268-274.
9. Carnes CA, Chung MK, Nakayama T, Nakayama H, Baliga RS, et al. (2001) Ascorbate attenuates atrial pacing-induced peroxynitrite formation and electrical remodeling and decreases the incidence of postoperative atria fibrillation. Circ Res 89: E32-E38. 